\title{
Simultaneous monitoring of chromatic dispersion and optical signal to noise ratio in optical links using convolutional neural network and asynchronous delay-tap sampling
}

\author{
Tomasz MROZEK*, Krzysztof PERLICKI, ANDRZEJ JAKUBIAK \\ Institute of Telecommunications, Warsaw University of Technology, \\ Nowowiejska 15/19, 00-665 Warsaw, Poland \\ ${ }^{*}$ Corresponding author: T.Mrozek@tele.pw.edu.pl
}

\begin{abstract}
The article presents a method for image analysis using asynchronous delay-tap sampling (ADTS) technique and convolutional neural networks (CNNs), allowing simultaneous monitoring of many phenomena occurring in the physical layer of the optical network. The ADTS method makes it possible to visualize the course of the optical signal in the form of characteristics (so-called phase portraits), which change their shape under the influence of phenomena (including chromatic dispersion, amplified spontaneous emission noise and other). Using the VPIphotonics software, a simulation model of the ADTS technique was built. After the simulation tests, 10000 images were obtained, which after proper preparation were subjected to further analysis using $\mathrm{CNN}$ algorithms. The main goal of the study was to train a CNN to recognize the selected impairment (distortion); then to test its accuracy and estimate the impairment for the selected set of test images. The input data consisted of processed binary images in the form of two-dimensional matrices, with the position of the pixel. This article focuses on the analysis of images containing simultaneously the phenomena of chromatic dispersion and optical signal to noise ratio.
\end{abstract}

Keywords: deep learning, convolutional neural networks, chromatic dispersion, OSNR, asynchronous delay-tap sampling.

\section{Introduction}

According to the guidelines of the International Telecommunication Union (ITU), the most important parameters in the design of optical networks are chromatic dispersion (CD) and polarization mode dispersion (PMD), optical signal to noise ratio (OSNR), crosstalk and attenuation. For the management, monitoring and diagnostics of optical connections, CD, PMD and OSNR are the most measurable key parameters. The parameters of the $\mathrm{CD}$ and PMD are crucial during the modernization of the network, enabling proper tuning of the devices or increasing the range. OSNR is in turn, important in designing 
$\mathrm{T}$ a b l e 1. The level of accuracy required for the parameter being monitored [1].

\begin{tabular}{ll}
\hline Monitored parameter & Accuracy required \\
\hline CD & better than $\pm 2 \%$ \\
PMD & better than $\pm 2 \%$ \\
OSNR & better than $\pm 0.5 \mathrm{~dB}$ \\
\hline
\end{tabular}

and maintaining the correct operation of optical links, because its value clearly translates into the level of errors in the channel. Table 1 presents required levels of accuracy for key monitored parameters.

These phenomena occur in the physical layer of the optical network. To be able to ensure the proper quality and correctness of transmission, they should be constantly monitored. Phenomena may occur simultaneously, therefore it is important to provide an appropriate method that will allow simultaneous monitoring and determination of individual disturbance values. Early techniques allowed for the measurement of these phenomena, but to a limited extent and in an individual way for each phenomenon. This involved the necessity of using several methods at the same time, which resulted in an increase in the operating costs and maintenance of the monitoring system as well as an increase in the complexity of the measurement system. Along with the development of optical networks, new methods have been proposed and introduced, which by their action began to include simultaneous monitoring of many phenomena. The following methods are currently used to monitor parameters in the physical layer: eye chart, methods based on monitoring of the signal subcarrier, asynchronous amplitude histogram technique, techniques of measuring relative delay between sidebands, parametric asynchronous eye diagram, asynchronous delay-tap sampling (ADTS). Each of the mentioned methods is characterized by different properties and limitations [2 $-\underline{5}$ ]. Very good properties are characterized by asynchronous techniques, which are char-

$\mathrm{T}$ a b l e 2. Summary of asynchronous delay-tap sampling techniques [్].

\begin{tabular}{|c|c|c|}
\hline Data analysis techniques & Formats demonstrated & Impairments demonstrated (range) \\
\hline $\begin{array}{l}\text { Support vector machine } \\
{[\underline{3}, \underline{4}, \underline{6}, \underline{7}]}\end{array}$ & $\begin{array}{l}\text { 10G NRZ, } \\
\text { 40G NRZ-DPSK, } \\
\text { 40G RZ-DQPSK, } \\
\text { 80G PM-DQPSK }\end{array}$ & $\begin{array}{l}\text { OSNR }(10,30) \mathrm{dB}, \\
\mathrm{CD}(-1400,1400) \mathrm{ps} / \mathrm{nm}, \\
\text { PMD }(0,60) \mathrm{ps}, \\
\text { In-band crosstalk }(15,25) \mathrm{dB}, \\
\text { Filter offset }(-12,12) \mathrm{GHz}\end{array}$ \\
\hline $\begin{array}{l}\text { Hough transformation } \\
{[\underline{8}-\underline{10}]}\end{array}$ & $\begin{array}{l}\text { 10G RZ-DPSK, } \\
\text { 20G RZ-DQPSK, } \\
\text { 40G NRZ-DPSK }\end{array}$ & $\begin{array}{l}\text { OSNR }(8.7,35) \mathrm{dB}, \\
\mathrm{CD}(-600,600) \mathrm{ps} / \mathrm{nm}\end{array}$ \\
\hline $\begin{array}{l}\text { Homodyne detection } \\
{[\underline{11}]}\end{array}$ & 10G NRZ-DPSK & $\begin{array}{l}\text { OSNR }(10,30) \mathrm{dB}, \\
\mathrm{CD}(0,800) \mathrm{ps} / \mathrm{nm}\end{array}$ \\
\hline $\begin{array}{l}\text { Hausdorff measure } \\
{[\underline{12}]}\end{array}$ & $\begin{array}{l}\text { 10G NRZ OOK, } \\
\text { 40G NRZ-DPSK }\end{array}$ & $\mathrm{CD}(0,400) \mathrm{ps} / \mathrm{nm}$ \\
\hline $\begin{array}{l}\text { Artificial neural networks } \\
{[\underline{13}]}\end{array}$ & 10G NRZ & $\begin{array}{l}\text { OSNR }(15,30) \mathrm{dB}, \\
\mathrm{CD}(0,55) \mathrm{ps} / \mathrm{nm}, \\
\operatorname{PMD}(0,10) \mathrm{ps}\end{array}$ \\
\hline
\end{tabular}


acterized by the simplicity of measurement systems. The use of asynchronous methods allows to observe disturbances in the form of characteristics that change their shape depending on the phenomena that occur. Characteristics with interference should be further analyzed in order to determine their numerical values. Appropriate techniques for data analysis are required for this, the most popular are presented in Table 2.

Each of the presented data analysis techniques has limitations primarily in terms of measurability.

The paper presents research based on new and previously not investigated method that utilizes convolutional neural networks $(\mathrm{CNN})$. Next section describes asynchronous delay-tap sampling, CNNs and their application for predicting values of chromatic dispersion and OSNR.

\section{Background asynchronous delay-tap sampling}

The presented asynchronous delay-tap sampling (ADTS) method [ $\underline{3}]$ enables the direct measurement of signal distortion without the need of recovering the synchronization clock. In this method, the received signal is subjected to demodulation and converted from the optical to the electrical domain. Then, the electric signal is separated into two feed lines, one of which introduces inflict physical delay $\Delta t$. Thus, the delay line propagates a delayed copy of the original signal. Both the original and time-shifted signals are directed to the two inputs of the analyzer, in which the sampling process is being activated. Sampling results in a creation of coordinates for a single point (pairs $x_{1}, y_{1}$, etc.). The processed signals from two feed lines represent a pair of samples ( $x, y$ coordinates) and are shown in Fig. 1.

Red vertical lines in Fig. 2 show the moments of sampling of the signal. As one may observe, the samples are extracted from different locations of the waveform with the sampling period $T_{\mathrm{s}}$ that is not related to the rate of the signal being monitored. The sampling period can be adjusted across several orders of magnitude. The obtained sample pairs are used to create dotted plots on which sample values $x_{i}$ and $y_{i}$ are the coordinates of a particular point on the chart. The shape of the dot plots is disturbed by various signal

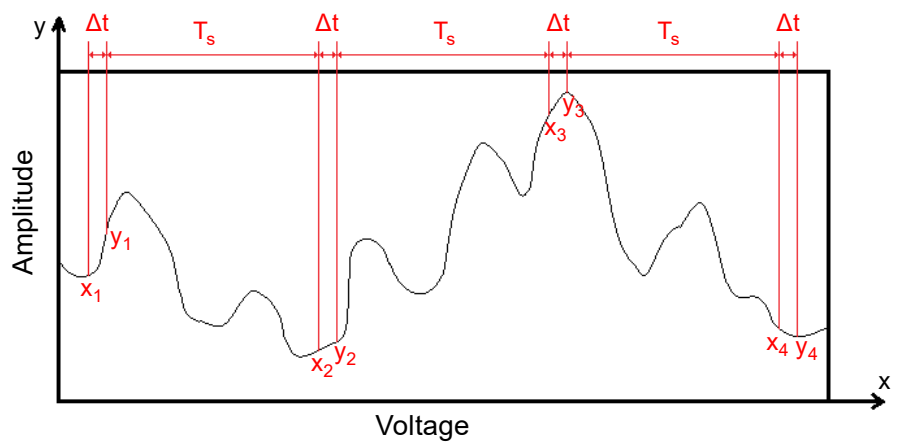

Fig. 1. A method of sampling the waveform in ADTS method, where $\Delta t$ - bit delay, $T_{\mathrm{s}}-$ sampling period, $\left(x_{i}, y_{i}\right)$ - pair of consecutive samples. 


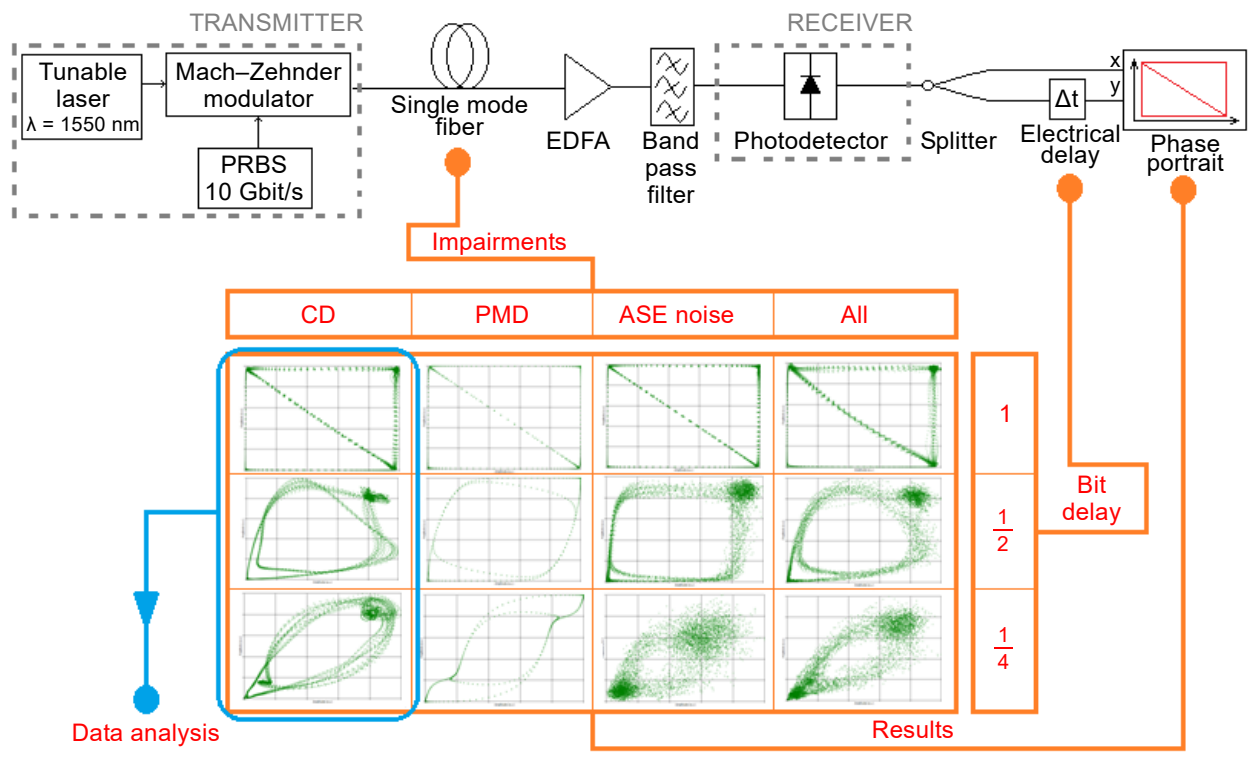

Fig. 2. Asynchronous delay-tap sampling - simulation model and impairments in physical layer.

parameters, and therefore the presented test method can be used to monitor among others: chromatic dispersion, polarization mode dispersion, and OSNR [14]. Methods based on the asynchronous delay-tap sampling allow to monitor signals of different bit rates and modulation formats. The measurement system is not simple, because it requires a precise matching of the delay $\Delta t$ used in the delay line. This matching is done based on the second sample pair. Nevertheless, the significant advantage of this method is its asynchronous capability that makes the recovery of the synchronization clock in the receiver part unnecessary $[\underline{3}, \underline{15}]$.

In the ADTS method it is significant that each parameter has a different effect on the shape of the dotted plots. For a $\Delta t$ delay the following distortions may be observed: chromatic dispersion bends the upper right edge of the plot to the center, ASE noise causes the blur of the edges of the plot, DGD characteristically rounds up the upper left and lower right corners of the plot.

The described simulation method was carried out using VPIphotonics software. The discussed test case scenario was carried out for the bit rate of $10 \mathrm{Gbit} / \mathrm{s}$, non-return -to-zero (NRZ) coding, and a wavelength of $1550 \mathrm{~nm}$. The simulation model and the type of interference, delays and bit sample portraits phases are shown in Fig. 2.

\section{Background convolutional neural networks}

In the proposed approach, we use machine learning methods for providing predictions about particular parameters in optical performance monitoring systems. In principle, information about chromatic dispersion and optical signal-to-noise ratio may be retrieved from simple signal representations such as delay-tap plots. It is desired to have 
methods for automated detection of certain impairments in the optical system. In data science we refer to this kind of tasks as classification problems. Moreover, such system may not only classify certain optical phenomena, but also provides qualitative information about them. Such task is a problem of regression - providing a qualitative prediction about a certain feature. In this work we focus on providing predictions about the chromatic dispersion and OSNR based on delay-tap plots. We used convolutional neural networks (CNNs) as our regression model for this problem.

\subsection{Convolutional neural networks}

Convolutional neural networks (CNNs) are artificial neural networks, which use the convolution operation to process high-dimensional inputs such as images. The convolution is basically defined for two continuous functions $f: \mathrm{R} \rightarrow \mathrm{R}$ and $g: \mathrm{R} \rightarrow \mathrm{R}$ as follows:

$$
(f * g)(t)=\int_{-\infty}^{+\infty} f(\tau) g(t-\tau) \mathrm{d} \tau
$$

where $f * g$ denotes convolution of functions $f$ and $g$, see, e.g., BRACEWELL [16], SMITH [17]. The convolution has regular algebraic properties, e.g., it is commutative, associative, distributive over addition and associative with scalar multiplication. In many areas of applications, such as signal processing or computer vision, the convolution of discrete functions is used:

$$
(f * g)(n)=\sum_{m=-\infty}^{\infty} f(m) g(n-m)
$$

where $f: \mathrm{Z} \rightarrow \mathrm{Z}$ and $g: \mathrm{Z} \rightarrow \mathrm{Z}$.

In image processing, where images can be represented as matrices of pixels, the convolution of two dimensional discrete functions is used:

$$
(f * g)(m, n)=\sum_{r=-\infty}^{\infty} \sum_{s=-\infty}^{\infty} f(r, s) g(m-r, n-s)
$$

where $f: \mathrm{Z} \times \mathrm{Z} \rightarrow \mathrm{Z}$ and $g: \mathrm{Z} \times \mathrm{Z} \rightarrow \mathrm{Z}$.

In our situation, a black-and-white image is represented as matrix of pixels $M_{m, n}$ where $m_{i, j}$ represents a particular pixel and $m_{i, j} \in\{0,1, \ldots, 254,255\}$, i.e., elements of matrix $M_{m, n}$ represent shades of grey (where 0 stands for black and 255 for white). Such image is represented by two dimensional function $h: Z_{m} \times Z_{n} \rightarrow Z_{256}$, where $Z_{k}:=\{0,1, \ldots, k-1\}$. In image processing very often one of the convoluted images is much smaller then another and is used as a filter, therefore, in fact, the commutativity of convolution is not needed. Thus in image processing, a convolution of images is often defined as matrix multiplication (which, in general, is not commutative).

CNNs were first introduced by LECUN et al. [18]. The convolution operation uses several feature maps (filters), by which a portion of the pixel map is processed. Those 


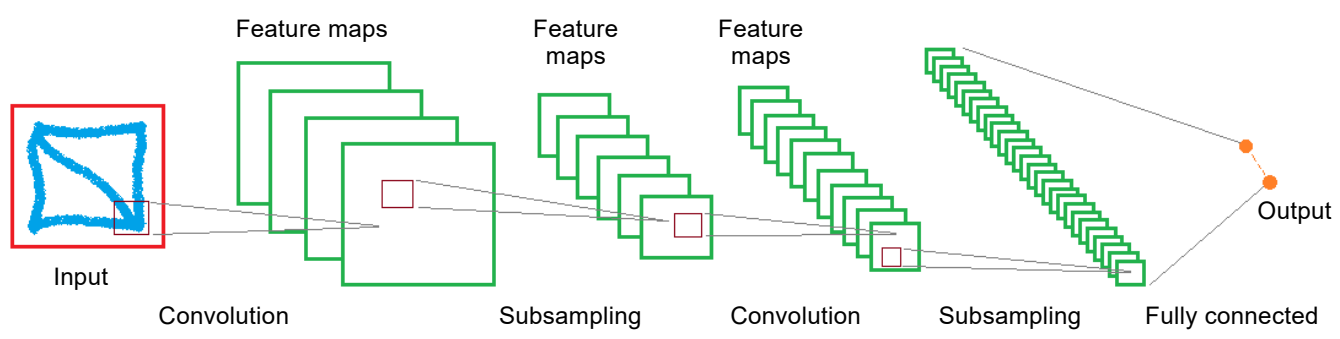

Fig. 3. Example of convolutional neural networks schema (source: based on Wikipedia).

feature maps are learned by the network. Next, the outputs of those filters are subsampled and provided to the next convolutional layer. This process may be repeated several times. The output of the last convolutional layer is provided to a fully connected layer of neurons, and finally to the output of the network. CNNs provide superior performance in image recognition tasks such as handwriting recognition or medical image analysis. Figure 3 presented an example of architecture of a CNN.

\subsection{Data preprocessing}

The ADTS methods provides a stream of $(x, y)$ pairs, where $x$ is the sampled value of the non-delayed signal and $y$ is the sampled value of the $1-$, or $1 / 2-$, or $1 / 4$-bit delayed signal. The delay-tap plots are basically plots generated from a $N$-element series of $(x, y)$ pairs. CNN uses images as the input data, which are 2-dimensional matrices of values of shape $W \times H$, where $W, H$ are the width and height of the image, respectively. In our approach, we will use input images of size $100 \times 100$.

To generate the input matrices, we use the following procedure. First we take $N$ $(x, y)$-pairs and rescale all pairs such that $0<x<W$ and $0<y<H$. Then we quantize $x$ and $y$ values to the natural numbers by calculating the floor value of each. Finally, we create a $W \times H$ matrix $M$ filled with zeros; for each processed $(x, y)$ in our list we set $M(x, y)$ to 1 . The $M$ matrix contains the representation of our delay-tap plot.

For training our regression model, a dataset containing many examples is needed. The examples in such dataset must contain pairs of input values and corresponding tar-

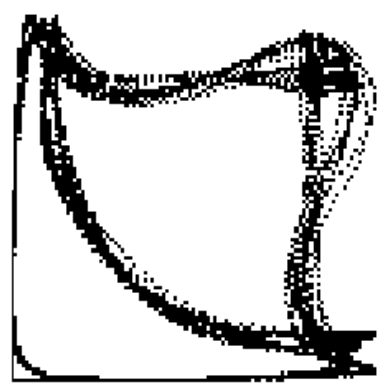

Fig. 4. Example input from dataset which shows 1 bit delay in images and $100 \times 100$ image resolution which were used in the experiment. 
get values. In our case, as we want to predict dispersion and its value and ASE noise and its value, such pairs will consist of a matrix $M$, representing the delay-tap plot, as the input value; and the corresponding chromatic dispersion and ASE noise as the output values.

For experiments we created one dataset for size of the delay-tap plot $100 \times 100$ and for $\Delta t=1$ bit-delay. Dataset consists of 10000 examples, which were created for CD values ranging from 0 to $2000 \mathrm{ps} / \mathrm{nm}$ and OSNR values ranging from 10 to $40 \mathrm{~dB}$. The example input image from dataset is shown in Fig. 4.

\subsection{Model and setting network parameters}

Regression model using convolutional neural networks was built. The implementation was based on TensorFlow [19] and TFLearn libraries. We used a de facto standard architecture of our network: the first two layers were convolutional layers with max pooling. We use 32 filters of size $3 \times 3$ and 64 filters of size $3 \times 3$, for the first and second layer, respectively. Both layers use rectified linear units (ReLu). Next, there are two fully connected layers using tanh activation for both 512 units, respectively. The output layer is a fully connected layer with one linear unit, that provides the output value. The model was trained using "Adam" optimizer for a root mean square error (RMSE) as the loss function. The remaining learning parameters were as follows: learning rate $=0.0001$, batch size respectively: 200,150,100,50 and 25. We split the dataset into training sets and verification sets with ratio: $90 \%$ for training, and $10 \%$ for verification. For both impairments (CD and OSNR) the network was trained in epochs range from 1 to 45 . That is 225 combinations for each phenomenon. Figure 5 presents a scheme for recognizing simultaneously occurring phenomena in the physical layer of the optical network.

After learning the network for all 225 combinations for each phenomenon, the best two models were selected. The coefficient of determination $R^{2}$ was used to de-

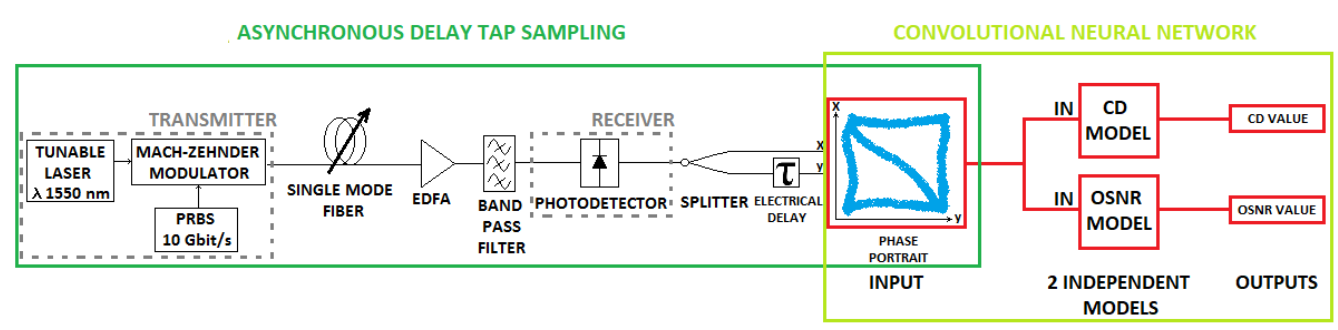

Fig. 5. Scheme for simultaneous recognition of occurring phenomena.

T a b l e 3. Properties of selected CNN models for CD and OSNR.

\begin{tabular}{lll}
\hline Impairments & CD & OSNR \\
\hline Epoch (number of pass over the full training set) & 36 & 41 \\
Batch size (number of training examples in one forward/backward pass) & 50 & 25 \\
Coefficient of determination $R^{2}$ & 0.997 & 0.989 \\
\hline
\end{tabular}


termine the best model. This is one of the basic measures of the quality of model fitting. The $R^{2}$ takes values from 0 to 1 . The models match is better when $R^{2}$ value is closer to one. For values in the range from 0.9 to 1 , the fit of the model is very good [20]. Table 3 presents the most important properties of selected models. For the CD model, the coefficient of determination is 0.997 , and for OSNR model, $R^{2}$ is 0.989 .

Section 4 presents examples of the results of recognizing simultaneously occurring impairments using the presented CNN models.

\section{Results and discussion}

The generated samples in the current work focused on the analysis of the chromatic dispersion impairment (CD) and optical signal to noise ratio (OSNR), which were
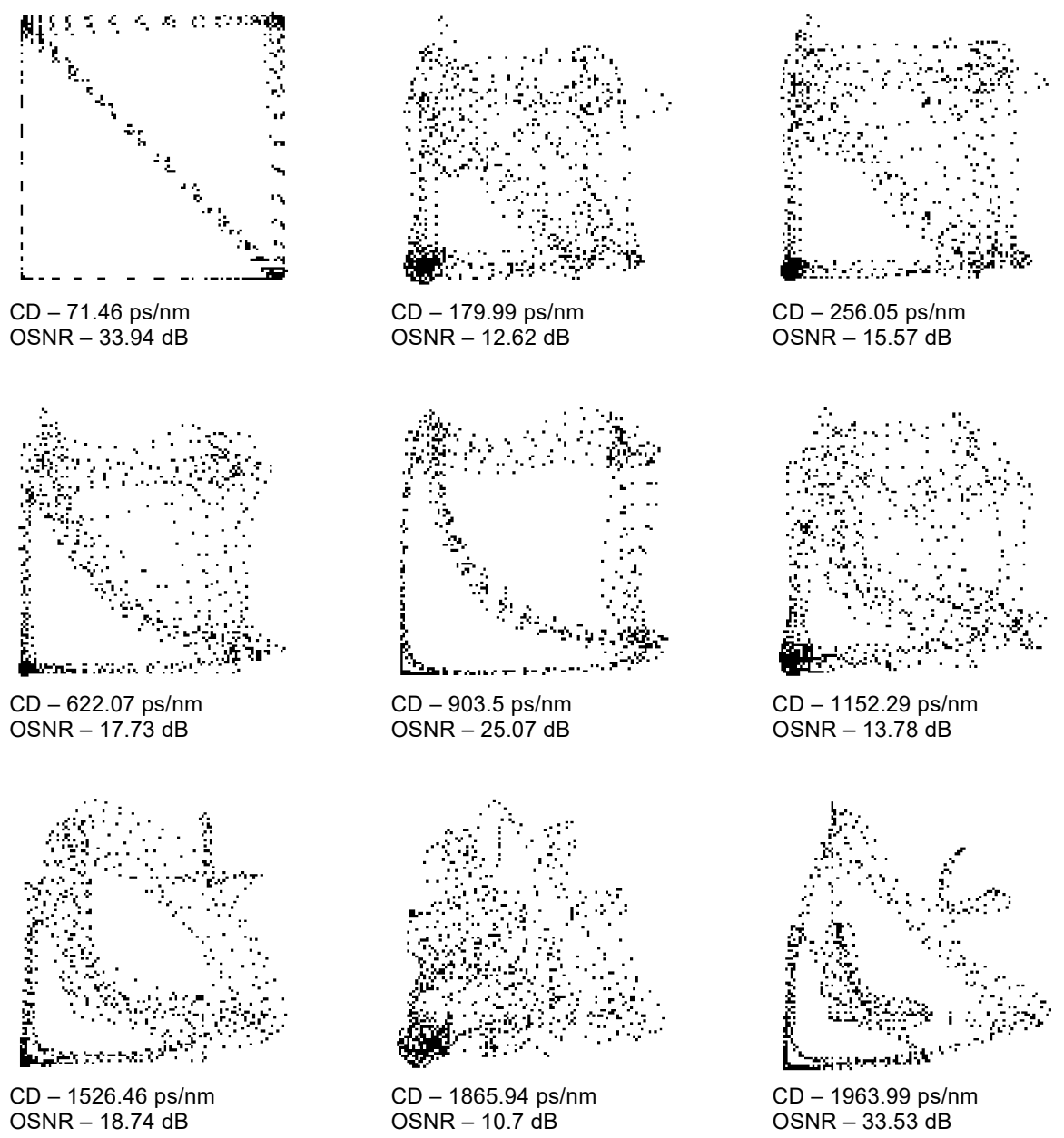

Fig. 6. Sample images of the test set and their actual impairments. 
measured in the range of 0 to $2000 \mathrm{ps} / \mathrm{nm}$ for $\mathrm{CD}$, and 10 to $40 \mathrm{~dB}$ for OSNR. Figure 6 presents sample phase portraits from the test set and their actual impairments.

In subsections 4.1 and 4.2, the results of impairments estimation for randomly selected samples are presented. In addition, Tables 4 and 5 also contain the results of estimating the values of CD and OSNR for the phase portraits presented in Fig. 6.

\subsection{Chromatic dispersion}

Table 4 presents the results of $\mathrm{CD}$ estimation values $\left(\mathrm{CD}_{\mathrm{E}}\right)$ and $\mathrm{CD}$ estimation errors values $\left(\mathrm{CD}_{\mathrm{F}-\mathrm{E}}\right)$ for selected chromatic dispersion $\left(\mathrm{CD}_{\mathrm{F}}\right)$.

\subsection{Optical signal to noise ratio}

Table 5 presents the results of OSNR estimation values $\left(\mathrm{OSNR}_{\mathrm{E}}\right)$ and $\mathrm{OSNR}$ estimation errors values $\left(\mathrm{OSNR}_{\mathrm{F}-\mathrm{E}}\right)$ for selected optical signal to noise ratio $\left(\mathrm{OSNR}_{\mathrm{F}}\right)$.

The results of the estimation of the impairments values obtained from the combination and application of the ADTS and CNN methods are very good. The estimation errors of the CD and OSNR parameters are within the required accuracy shown in Table 1. With a successful result, a large measuring range of simultaneously occurring impairments, from 0 to $2000 \mathrm{ps} / \mathrm{nm}$ for CD and from 10 to $40 \mathrm{~dB}$ for OSNR were tested. Figure 6 shows examples of phase portraits, with different levels of disturbance by given phenomena. Convolutional neural networks perform very well in recognizing the value of impairments, regardless of the strength of a given phenomenon, which is confirmed by data presented in Tables 4 and 5. This data also include the results of estimation of phenomena occurring in phase portraits in presented Fig. 6.

$\mathrm{T}$ a b $1 \mathrm{e}$ 4. Results of CD prediction error.

\begin{tabular}{|c|c|c|c|c|c|c|c|}
\hline \multirow{2}{*}{$\begin{array}{l}\text { Sample } \\
\text { number }\end{array}$} & $\mathrm{CD}_{\mathrm{F}}$ & $\mathrm{CD}_{\mathrm{E}}$ & $\mathrm{CD}_{\mathrm{F}-\mathrm{E}}$ & \multirow{2}{*}{$\begin{array}{l}\text { Sample } \\
\text { number }\end{array}$} & $\mathrm{CD}_{\mathrm{F}}$ & $\mathrm{CD}_{\mathrm{E}}$ & $\mathrm{CD}_{\mathrm{F}-\mathrm{E}}$ \\
\hline & \multicolumn{3}{|c|}{$[\mathrm{ps} / \mathrm{nm}]$} & & \multicolumn{3}{|c|}{$[\mathrm{ps} / \mathrm{nm}]$} \\
\hline 1 & 71.46 & 70.25 & 1.21 & $\overline{16}$ & 1010.93 & 1006.87 & 4.05 \\
\hline 2 & 179.99 & 181.20 & 1.21 & 17 & 1018.49 & 1014.31 & 4.18 \\
\hline 3 & 256.05 & 258.66 & 2.62 & 18 & 1055.07 & 1046.41 & 8.66 \\
\hline 4 & 388.92 & 393.18 & 4.25 & 19 & 1152.29 & 1173.20 & 20.91 \\
\hline 5 & 397.63 & 403.47 & 5.84 & 20 & 1278.40 & 1286.89 & 8.49 \\
\hline 6 & 539.26 & 543.67 & 4.41 & 21 & 1441.46 & 1440.31 & 1.15 \\
\hline 7 & 622.07 & 623.24 & 1.17 & 22 & 1486.89 & 1480.73 & 6.15 \\
\hline 8 & 711.42 & 721.04 & 9.62 & 23 & 1526.46 & 1528.90 & 2.44 \\
\hline 9 & 738.95 & 753.03 & 14.08 & 24 & 1594.66 & 1583.27 & 11.40 \\
\hline 10 & 747.28 & 752.75 & 5.48 & 25 & 1677.84 & 1672.11 & 5.73 \\
\hline 11 & 809.68 & 816.03 & 6.35 & 26 & 1719.57 & 1726.12 & 6.55 \\
\hline 12 & 828.85 & 840.09 & 11.24 & 27 & 1801.32 & 1803.51 & 2.19 \\
\hline 13 & 903.50 & 903.57 & 0.08 & 28 & 1865.94 & 1862.03 & 3.91 \\
\hline 14 & 941.44 & 936.02 & 5.42 & 29 & 1908.91 & 1922.35 & 13.43 \\
\hline 15 & 998.13 & 1001.98 & 3.84 & 30 & 1963.99 & 1968.12 & 4.13 \\
\hline
\end{tabular}


T a b l e 5. Results of OSNR prediction error.

\begin{tabular}{|c|c|c|c|c|c|c|c|}
\hline \multirow{2}{*}{$\begin{array}{l}\text { Sample } \\
\text { number }\end{array}$} & OSNR $_{\mathrm{F}}$ & $\mathrm{OSNR}_{\mathrm{E}}$ & $\mathrm{OSNR}_{\mathrm{F}-\mathrm{E}}$ & \multirow{2}{*}{$\begin{array}{l}\text { Sample } \\
\text { number }\end{array}$} & $\mathrm{OSNR}_{\mathrm{F}}$ & \multirow{2}{*}{$\begin{array}{r}\mathrm{OSNR}_{\mathrm{E}} \\
{[\mathrm{dB}]}\end{array}$} & \multirow[t]{2}{*}{$\mathrm{OSNR}_{\mathrm{F}-\mathrm{E}}$} \\
\hline & \multicolumn{3}{|c|}{$[\mathrm{dB}]$} & & & & \\
\hline 1 & 10.4315 & 10.3985 & 0.0329 & 16 & 20.3386 & 20.2669 & 0.0717 \\
\hline 2 & 10.7039 & 10.6688 & 0.0351 & 17 & 23.2718 & 23.2814 & 0.0096 \\
\hline 3 & 12.6219 & 12.9876 & 0.3656 & 18 & 24.2721 & 24.2119 & 0.0602 \\
\hline 4 & 13.0533 & 13.0611 & 0.0078 & 19 & 24.5149 & 24.3527 & 0.1622 \\
\hline 5 & 13.7062 & 13.5996 & 0.1066 & 20 & 25.0706 & 25.0865 & 0.0159 \\
\hline 6 & 13.7799 & 13.7701 & 0.0098 & 21 & 26.5620 & 26.7739 & 0.2119 \\
\hline 7 & 13.8401 & 13.8333 & 0.0068 & 22 & 27.3333 & 27.3810 & 0.0476 \\
\hline 8 & 13.9505 & 14.0718 & 0.1213 & 23 & 28.3784 & 28.3735 & 0.0049 \\
\hline 9 & 14.7805 & 14.6910 & 0.0895 & 24 & 29.7163 & 29.5375 & 0.1788 \\
\hline 10 & 15.5720 & 15.5451 & 0.0269 & 25 & 30.5308 & 30.5147 & 0.0161 \\
\hline 11 & 17.7265 & 17.6698 & 0.0568 & 26 & 33.5329 & 33.3384 & 0.1945 \\
\hline 12 & 18.7407 & 18.6192 & 0.1215 & 27 & 33.9388 & 33.9448 & 0.0060 \\
\hline 13 & 18.8344 & 18.8724 & 0.0380 & 28 & 34.6571 & 34.5700 & 0.0871 \\
\hline 14 & 18.9429 & 18.8195 & 0.1234 & 29 & 37.3428 & 37.3979 & 0.0551 \\
\hline 15 & 19.8670 & 19.8265 & 0.0405 & 30 & 37.7410 & 37.4584 & 0.2826 \\
\hline
\end{tabular}

\section{Conclusion}

The article presents the use of ADTS and convolution neural networks techniques in the recognition and measurement of simultaneous impairments of chromatic dispersion and optical signal to noise ratio in the physical layer of the optical network. The results obtained are good. CNN models work well at identifying phenomena that occur simultaneously during transmission. At a later stage of the work, attempts will be made to teach the network in recognizing additional occurring impairments simultaneously like polarization mode dispersion (PMD) or crosstalk. Work will be undertaken to obtain better estimation accuracy for the measured impairments. This will require generating more samples. In addition, the impact of other network settings (number of epoch, batch size etc.) on the accuracy of fault recognition will be checked.

\section{References}

[1] Dahan D., Mahlab U., Teixeira A., Zacharopoulos I., Tomkos I., Optical performance monitoring for translucent/transparent optical networks, IET Optoelectronics 5(1), 2011, pp. 1-18, DOI: 10.1049/ iet-opt.2010.0010.

[2] Perlicki K., Investigation of the state of polarization distribution generated by polarization scramblers on the Poincarè sphere, Optics Communications 252(1-3), 2005, pp. 58-63, DOI: 10.1016/ j.optcom.2005.04.002.

[3] Dods S.D., Anderson T.B., Optical performance monitoring technique using delay tap asynchronous waveform sampling, 2006 Optical Fiber Communication Conference and the National Fiber Optic Engineers Conference, Anaheim, CA, 2006, DOI: 10.1109/OFC.2006.215890. 
[4] Anderson T.B., Kowalczyk A., Clarke K., Dods S.D., Hewit D., Li J.C., Multi impairment monitoring for optical networks, Journal of Lightwave Technology 27(16) 2009, pp. 3729-3736, DOI: 10.1109/JLT.2009.2025052.

[5] Anderson T.B., Dods S.D., Kowalczyk A., Clarke K., Hewitt D., Li J.C., Chapter 7 - Optical performance monitoring based on asynchronous delay-tap sampling, [In] Chan C.C.K., Optical Performance Monitoring: Advanced Techniques for Next-Generation Photonic Networks, Academic Press, 2010, pp. 175-192, DOI: 10.1016/B978-0-12-374950-5.00007-9.

[6] Clarke K., et al., Experimental demonstration of multi-impairment monitoring on a commercial $10 \mathrm{~Gb} / \mathrm{s}$ NRZ channel, Proc. OFC/NFOEC, San Diego, California, 2009, paper OThH7.

[7] Beaman D., et al., Demonstration of simultaneous OSNR and CD monitoring using asynchronous delay tap sampling on an $800 \mathrm{~km}$ WDM test bed, Proc., ECOC, Vienna, Austria, 2009, paper 9.3.4.

[8] Kozicki B., TakuYa O., Hidehío T., Optical performance monitoring of phase-modulated signals using asynchronous amplitude histogram analysis, Journal of Lightwave Technology 26(10), 2008, pp. 1353-1361, DOI: 10.1109/JLT.2008.917374.

[9] Kozicki B., Maruta A., Kitayama K., Experimental demonstration of optical performance monitoring for RZ-DPSK signals using delay-tap sampling method, Optics Express 16(6), 2008, pp. 3566-3576, DOI: $10.1364 / \mathrm{OE} .16 .003566$.

[10] Kitayama K., KozIcki B., Maruta A., Asynchronous optical performance monitoring of RZ DQPSK signals using delay-tap sampling, Proc. ECOC, Berlin, Germany, 2007, paper P060.

[11] Choi H.Y., Takushima Y., Chung Y.C., Multiple impairment monitoring technique using optical field detection and asynchronous delay-tap sampling method, Proc. OFC/NFOEC, San Diego, California, 2009, paper 0ThJ5.

[12] Zhaо J., Lu C., Lam K.M., Li Z., TAм H.Y., Wai P.K.A., A novel optical signal monitoring method of DPSK signal based on delay tap sampling and Hausdorff distance measure, Proc. CLEO/QELS, San Jose, California, 2008, paper JWA108.

[13] JARGON J.A., Wu X., Willner A.E., Optical performance monitoring using artificial neural networks with features derived from asynchronous delay tap sampling, Proc. CLEO/QELS, San Jose, California, 2009, paper OThH1.

[14] Willner A.E., Pan Z., Yu C., 7 - Optical performance monitoring, [In], Kaminow I. P., Li T., Willner A.E. [Eds.], Optical Fiber Telecommunications V B, 5th Ed., Academic Press, 2008, pp. 233-292, DOI: 10.1016/B978-0-12-374172-1.00007-2.

[15] Zhao J., Li Z., Liu D., Cheng L., Lu C., Tam H.Y., NRZ-DPSK and RZ-DPSK signals signed chromatic dispersion monitoring using asynchronous delay-tap sampling, Journal of Lightwave Technology 27(23), 2009, pp. 5295-5301, DOI: 10.1109/JLT.2009.2031610.

[16] Bracewell R., The Fourier Transform and Its Applications, 3rd Ed., McGraw-Hill, New York, 2000, pp. 24-54 and 331-334.

[17] Sмітн S.W., The Scientist and Engineer's Guide to Digital Signal Processing, 1st Ed., California Technical Publishing, 1997, pp. 246-252.

[18] LeCun Y., Bottou L., Bengio Y., Haffner P., Gradient-based learning applied to document recognition, Proceedings of the IEEE 86 (11), 1998, pp. 2278-2324, DOI: $10.1109 / 5.726791$.

[19] Abadi M., Agarwal A., Barham P., Brevdo E., Chen Z., Citro C., Corrado G.S., Davis A., Dean J., Devin M., Ghemawat S., Goodfellow I., Harp A., Irving G., Isard M., Jozefowicz R., Jia Y., Kaiser L., Kudlur M., Levenberg J., Mané D., Schuster M., Monga R., Moore S., Murray D., Olah C., Shlens J., Steiner B., Sutskever I., Talwar K., Tucker P., Vanhoucke V., Vasudevan V., Viégas F., Vinyals O., Warden P., Wattenberg M., Wicke M., Yu Y., Zheng X., TensorFlow: Large-scale machine learning on heterogeneous systems, 2015, Software available from tensorflow.org.

[20] Cameron A.C., Windmeijer F.A.G., An R-squared measure of goodness of fit for some common nonlinear regression models, Journal of Econometrics 77(2), 1997, pp. 329-342, DOI: 10.1016 / $\underline{\text { S0304-4076(96)01818-0. }}$. 